Research Article

\title{
Effect of the Time-Varying Damping on the Vibration Isolation of a Quasi-Zero-Stiffness Vibration Isolator
}

\author{
Xin Li $\mathbb{D}^{1,2}$ Jinqiu Zhang, ${ }^{1}$ and Jun Yao ${ }^{3}$ \\ ${ }^{1}$ Army Academy of Armored Forces, Beijing, China \\ ${ }^{2}$ Naval Research Academy, Beijing, China \\ ${ }^{3}$ Beijing Institute of Tracking and Telecommunications Technology, China \\ Correspondence should be addressed to Xin Li; lixin20170606@163.com
}

Received 13 January 2020; Accepted 16 April 2020; Published 8 May 2020

Academic Editor: Jean-Jacques Sinou

Copyright $(2020$ Xin Li et al. This is an open access article distributed under the Creative Commons Attribution License, which permits unrestricted use, distribution, and reproduction in any medium, provided the original work is properly cited.

\begin{abstract}
This study focuses on the effect of damping changes on the vibration isolation of a quasi-zero-stiffness vibration isolator. A nonlinearvibration equation for the quasi-zero-stiffness vibration isolator is found and solved using the multiscale method. Then, the vibration characteristics before, in the process of and after the damping change, are also examined. The results show that time-varying damping can be equivalent to the addition of a stiffness term to the vibration system, which leads to a change of the vibration amplitude frequency response, leakage of power spectrum, and corresponding linear spectrum features being weakened. When the damping changes rapidly, the vibration system tends to be divergent rather than stable. After the change, the number of stable focuses of the proposed quasi-zero-stiffness vibration isolator increases from one to two, and the system will see decline in its vibration stability.
\end{abstract}

\section{Introduction}

In a floating raft isolation system, the raft frame itself can effectively isolate high-frequency vibration, but plays a limited role in isolating low-frequency vibration. Since the low-frequency vibration travels long distance and is easy to detect, there has been extensive research on the measures to effectively reduce it. Among them, a quasi-zero-stiffness vibration isolator is needed for low-frequency vibration isolation through reducing the inherent frequency of the system. [1,2].

Quasi-zero-stiffness vibration isolators have been extensively studied as well. From the perspective of application, Valeev et al. designed a quasi-zero-stiffness vibration isolator for oil/gas transporters and analyzed the low-frequency vibration isolation performance [3]. From the perspective of vibration, Lan et al. designed and tested a kind of vibration isolator with a compact structure that can bear different masses [4]. Cheng et al. analyzed the vibration characteristics of a quasi-zero-stiffness vibration isolator at the primary resonance point and the $1 / 3$ resonance point under a constant external force. They found gradually softening properties near the primary resonance point and decreasing $1 / 3$ resonance band under the action of a constant external force. [5]. Xu et al. designed an electromagnetically adjustable quasi-zero-stiffness vibration isolator and studied its vibration isolation using both theoretical and experimental results. According to their study, the designed quasi-zero-stiffness isolator showed a higher vibration isolation efficiency than linear isolators [6]. Huang et al. examined the vibration isolation properties of a quasi-zero-stiffness isolator during vibration control [7]. Li et al. designed a device similar to a quasi-zero-stiffness isolator and examined its characteristics [8]. Kovacica et al. investigated the vibration isolation of a quasi-zero-stiffness isolator and analyzed the nonlinear-vibration features that may appear during the vibration process, such as bifurcation and chaos [9]. There are more examples, than the abovementioned, of those studies on the vibration isolation characteristics of quasi-zero-stiffness isolators, including such study in the context of underload/overload [10], subject to sinusoidal and stochastic excitations [11], and with a quasi-zero-stiffness isolator consisting of compound-shape memory alloys [12]. All of these studies focused on the effect of stiffness changes on the vibration isolation performance, which is in line with the main features of quasi-zero-stiffness isolators. However, damping is a parameter of equal 
importance to stiffness in a vibration system, which also requires detailed studies.

There has been very little research so far on the effect of damping changes on the vibration isolation performance of the quasi-zero-stiffness vibration isolator. Liu et al. examined the effect of nonlinear stiffness and nonlinear damping on the vibration isolation performance of a quasi-zerostiffness isolator [13]. Cheng et al. discussed the effect of nonlinear damping on the vibration transmissibility. The group concluded that nonlinear damping reduces the vibration transmissibility significantly [14]. Amabili derived accurately, for the first time, nonlinear damping from a fractional viscoelastic solid standard model by considering geometric nonlinearity [15]. Mofidian and Bardaweel focused on investigating the effect of nonlinear cubic viscous damping in a vibration isolation system, which consists of a magnetic spring with a positive nonlinear stiffness and a mechanical oblique spring with geometric nonlinear negative stiffness [16]. Even though the effect of damping was considered, damping was still only at a constant value in the abovementioned studies. In order to enhance the vibration isolation performance of the isolator against low-frequency vibration as much as possible, both damping and stiffness should be adjustable [17]. To this end, magnetorheological (MR) damper is the currently preferred option [18].

Magnetorheological fluid is "smart" material. Despite adjustable, the damping with this material is significantly influenced by temperature $[19,20]$. With the magnetorheological fluid as the absorber, when the magnetic field and the temperature field are in parallel, the heat transfer rate increases by $105 \%$ [21]. As the temperature exceeds $100^{\circ} \mathrm{C}$, the damping decreases rapidly [22]. When the fluid is used as a brake, the temperature should be strictly controlled to maintain the damping performance [23-25]. Likewise, magnetorheological elastomers have similar properties [26-29]. While previous research revealed a feature that damping is a variable as the temperature changes, rather than a constant, it did not consider this feature. To enhance the isolation performance of vibration isolators against low-frequency vibration, this study combines a quasi-zero-stiffness vibration isolator with a magnetorheological damper and analyzes the isolation performance of the quasi-zero-stiffness isolator against low-frequency vibration considering damping as a time-varying parameter.

\section{Vibration Model for a Quasi-Zero-Stiffness Vibration Isolator}

In this study, the designed quasi-zero-stiffness vibration isolator consists of two parts: a quasi-zero-stiffness isolator body and a magnetorheological damper (the dashed line part), whose structure is shown in Figure 1. Here, $m$ is the bearing mass, $k$ is the stiffness, $\delta$ is the nonlinear stiffness, $c(\tau)$ is the magnetorheological time-varying damping (where $\tau$ is the slow-scale time), $\xi$ is the nonlinear damping coefficient, $F$ is the excitation force amplitude, $\Omega$ is the excitation frequency, and $z$ is the vertical displacement of the rotor.

The mechanism of the vibration isolator can be described as follows. The vibrator moves vertically upon excitation. The magnetic teeth of the stator and the rotor produce a

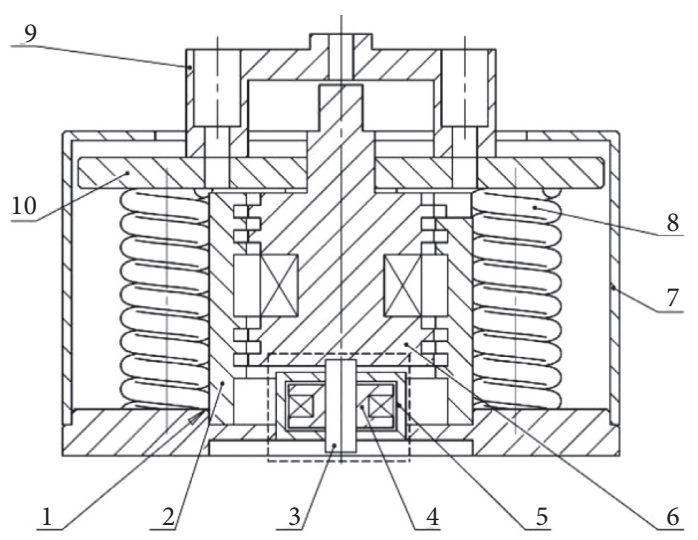

FIGURE 1: Structure of the proposed quasi-zero-stiffness vibration isolator: (1) base; (2) stator; (3) intermediate shaft; (4) valve body; (5) shell; (6) rotor (7) casing; (8) supporting spring; (9) support; (10) supporting plate.

relative displacement, the magnitude of the electromagnetic force changes, and the electromagnetic stiffness changes. The electromagnetic stiffness and spring stiffness together represent a nonlinear stiffness via a cubic term. The MR damper of the isolator provides damping. Due to current heating, damping changes over time.

Using Newton's Second Law of Motion, the vibration equation can be written as follows:

$$
m \frac{d^{2} z}{d t^{2}}+\frac{d[c(\tau) z]}{d t}+\xi\left(\frac{d z}{d t}\right)^{3}+k z+\delta z^{3}=F \cos (\Omega t)
$$

Since the slow-varying time can be formulated as $\tau=\varepsilon t$, where $\varepsilon$ is the perturbation parameter, the following expression can be derived: $(d / d t)=(d / d \tau)(d \tau / d t)=\varepsilon(d / d \tau)$. Accordingly, equation (1) is simplified to

$$
m \ddot{z}+c(\tau) \dot{z}+\varepsilon c(\tau) z+\xi(\dot{z})^{3}+k z+\delta z^{3}=F \cos (\Omega t),
$$

where $\ddot{z}=\left(d^{2} z / d t^{2}\right)$ and $\dot{z}=(d z / d t)$.

By comparing the vibration equation with a constant damping, the vibration equation of the quasi-zero-stiffness vibration isolator using time-varying damping is added with a stiffness item $\varepsilon c(\tau) z$, and the stiffness coefficient is a function of time-varying damping $\varepsilon c(\tau)$. As the damping changes, the inherent vibrational frequency changes.

Through further processing, equation (2) can be rewritten as follows:

$$
\ddot{z}+2 \varsigma w \dot{z}+2 \mu w(\dot{z})^{3}+(2 \varepsilon \dot{\zeta}+1) w z+\beta z^{3}=f \cos (\Omega t) .
$$

Here, $\varsigma=(c(\tau) / 2 w m), \dot{\zeta}=(c(\tau) / 2 w m), \mu=(\xi / 2 w m)$, $w^{2}=(k / m), \beta=(\delta / m)$, and $f=(F / m)$.

2.1. Solution to the Equation with a Constant Damping. When the damping is constant, the abovementioned equation can be solved using the multiscale method. Since equation (3) cannot be solved accurately adopting a 
numerical method, this study uses the multiscale method, introduces a small perturbation parameter $\mathcal{E}$, and performs the following scale transformation:

$$
\begin{aligned}
& \varsigma \longrightarrow \varepsilon \varsigma, \\
& \mu \longrightarrow \varepsilon \mu, \\
& \beta \longrightarrow \varepsilon \beta, \\
& f \longrightarrow \varepsilon f .
\end{aligned}
$$

By substituting equation (4) into equation (3) and retaining $\varepsilon^{0}$ and $\varepsilon^{1}$, the following expression can be derived:

$$
\ddot{z}+2 \varepsilon \varsigma w \dot{z}+2 \varepsilon \mu w(\dot{z})^{3}+(2 \varepsilon \zeta+1) w z+\varepsilon \beta z^{3}=\varepsilon f \cos (\Omega t) .
$$

Using the multiscale method, it was assumed that the solution to the equation could be expressed as follows:

$$
z(t, \varepsilon)=z_{0}\left(T_{0}, T_{1}\right)+\varepsilon z_{1}\left(T_{0}, T_{1}\right)+\mathrm{O}\left(\varepsilon^{2}\right) .
$$

Here, $T_{0}$ is the fast-time scale $\left(T_{0}=t\right)$ and $T_{1}$ is the slowtime scale $\left(T_{1}=\varepsilon t\right)$.

Near the quasi-zero-stiffness resonance point, the excitation frequency is

$$
\Omega=w+\varepsilon \sigma .
$$

Here, $\sigma$ is the adjusting parameter that causes $\Omega$ to approach $w$.

The differential operator can be written as follows:

$$
\begin{aligned}
\frac{\mathrm{d}}{\mathrm{d} t}(\cdot) & =\left(D_{0}+\varepsilon D_{1}\right)(\cdot), \\
\frac{\mathrm{d}^{2}}{\mathrm{~d} t^{2}}(\cdot) & =\left(D_{0}^{2}+2 \varepsilon D_{0} D_{1}\right)(\cdot), \\
D_{0} & =\frac{\partial}{\partial T_{0}}, \\
D_{1} & =\frac{\partial}{\partial T_{1}} .
\end{aligned}
$$

By substituting equations (6) to (8a) and (8b) into equation (5) and comparing the same-order coefficients of $\varepsilon$ for both two sides, the following expression can be derived:

$$
\begin{aligned}
\mathcal{E}^{0}: D_{0}^{2} z_{0}+w^{2} z_{0}= & 0, \\
\varepsilon^{1}: D_{0}^{2} z_{1}+w^{2} z_{1}= & -2 D_{0} D_{1} z_{0}-2 \varsigma w D_{0} z_{0}-2 \mu w\left(D_{0} z_{0}\right)^{3} \\
& -2 \varsigma w z_{0}-\beta\left(z_{0}\right)^{3}+f \cos \left(\Omega T_{0}\right) .
\end{aligned}
$$

The general solution to equation (9) can be written as follows:

$$
z_{0}=A e^{i w T_{0}}+\bar{A} e^{-i w T_{0}}
$$

where $A$ is a function of slow time $T_{1}$ and $\bar{A}$ is the conjugate complex of $A$.

By substituting equation (11) into equation (10) and eliminating the secular term, the following expression can be acquired:

$$
\begin{aligned}
- & 2 A^{\prime} i w-2 \varsigma w A i w-6 \mu w i A^{2} \bar{A} w^{3}-2 \zeta w A \\
& -3 \beta A^{2} \bar{A}+\frac{1}{2} f e^{i \sigma T_{1}}=0 .
\end{aligned}
$$

$A$ can be rewritten in the following polar form:

$$
A=\frac{1}{2} a e^{i \theta} .
$$

Here, the real numbers $a$ and $\theta$ are functions of slow time $T_{1}$.

By substituting equation (13) into equation (12) and separating the real part from the imaginary part, the following expressions can be obtained:

$$
\begin{gathered}
a^{\prime}=h_{1} a+h_{2} a^{3}+h_{5} \sin \gamma, \\
a \gamma^{\prime}=h_{3} a+h_{4} a^{3}+h_{5} \cos \gamma .
\end{gathered}
$$

Here, $\quad h_{1}=-w \varsigma, \quad h_{2}=-(3 / 4) \mu w^{3}, \quad h_{3}=-\sigma+\dot{\zeta}$, $h_{4}=(3 / 8)(\beta / w), h_{5}=-(1 / 2)(f / w)$, and $\gamma=\theta-\sigma T_{1}$.

2.2. Solution to the Equation with Varying Damping. In the process of damping changes, multiscaling is no longer applicable. The Runge-Kutta method is used to solve the amplitude frequency characteristic equation of quasi-zerostiffness isolator.

\section{Stability Analysis of the Solution}

3.1. Amplitude Frequency Response and the Solution's Stability Region. Let $a^{\prime}=0$ and $\gamma^{\prime}=0$ in equations (14a) and (14b), and the solution corresponding to the stable state of the quasi-zero-stiffness vibration isolator can be obtained. Equations (14a) and (14b) can then be simplified as follows:

$$
h_{5}^{2}-\left(h_{1} a+h_{2} a^{3}\right)^{2}-\left(h_{3} a+h_{4} a^{3}\right)^{2}=0 .
$$

There may be one or three solutions to equation (15). In the case of three solutions, a saddle-node bifurcation appeared and the frequency response curves jump during the vibration. In the case of only one solution, the critical amplitude can be solved as follows:

$$
h_{5 \_s t a b l e}=\sqrt{\frac{8}{27\left(h_{2}^{2}+h_{4}^{2}\right)^{2}}\left(h_{1} h_{4} \frac{h_{2}-\sqrt{3} h_{4}}{h_{4}+\sqrt{3} h_{2}}-h_{1} h_{2}\right)^{3}} .
$$

According to equation (16), the critical excitation amplitude is related to damping coefficient and stiffness.

The stability of the stationary vibration solution of the quasi-zero-stiffness vibration isolator can be treated as the stability of the autonomous system at the singular point $(\bar{a}, \bar{r})$. Therefore, the system can be treated as a linear system.

By linearizing equations (14a) and (14b) at the singular point $(\bar{a}, \bar{r})$, the autonomous differential equations of the disturbing quantities $\Delta \bar{a}$ and $\Delta \bar{r}$ can be written as follows: 


$$
\begin{gathered}
\frac{\mathrm{d} \Delta \bar{a}}{\mathrm{~d} T_{1}}=h_{1} \Delta \bar{a}+3 h_{2} \bar{a}^{2} \Delta \bar{a}+h_{5} \cos \bar{\gamma} \Delta \bar{\gamma}, \\
\bar{a} \frac{\mathrm{d} \Delta \bar{\gamma}}{\mathrm{d} T_{1}}=h_{3} \Delta \bar{a}+3 h_{4} \bar{a}^{2} \Delta \bar{a}-h_{5} \sin \bar{\gamma} \Delta \bar{\gamma} .
\end{gathered}
$$

Considering that $a \prime=\gamma^{\prime}=0, \bar{\gamma}$ in equations (17a) and (17b) can be eliminated and the characteristic equation can be written as follows:

$$
\operatorname{det}\left[\begin{array}{cc}
h_{1}+3 h_{2} \bar{a}^{2}-\lambda & N \\
M & h_{1}+h_{2} \bar{a}^{2}-\lambda
\end{array}\right]=0,
$$

where $M=(1 / \bar{a})\left[h_{3}+3 h_{4} \bar{a}^{2}\right]$ and $N=-\bar{a}\left[h_{3}+h_{4} \bar{a}^{2}\right]$.

Expanding equation (18) yields

$$
\begin{aligned}
\lambda^{2} & -\left(2 h_{1}+4 h_{2} \bar{a}^{2}\right) \lambda+h_{1}{ }^{2}+h_{3}{ }^{2}+4\left(h_{1} h_{2}+h_{3} h_{4}\right) \bar{a}^{2} \\
& +3\left(h_{2}{ }^{2}+h_{4}{ }^{2}\right) \bar{a}^{4}=0 .
\end{aligned}
$$

In the case of $2 h_{1}+4 h_{2} \bar{a}^{2}<0$, the instability condition of the stationary solution can be written as follows:

$$
h_{1}^{2}+h_{3}^{2}+4\left(h_{1} h_{2}+h_{3} h_{4}\right) \bar{a}^{2}+3\left(h_{2}{ }^{2}+h_{4}{ }^{2}\right) \bar{a}^{4}<0 \text {. }
$$

The stable region of the solution can now be evaluated according to equation (20).

3.2. Stability of the Bifurcation Solution. Excitation only changes the position of the dynamic bifurcation equilibrium point for a slow-time scale $\tau$, during which the bifurcation properties remain unchanged. Therefore, the effect of the excitation is not considered when investigating the dynamic bifurcation properties of the quasi-zero-stiffness vibration isolators. Let $h_{5}=0$, and equations (14a) and (14b) can be rewritten as follows:

$$
\begin{aligned}
& a^{\prime}=h_{1} a+h_{2} a^{3}, \\
& \gamma^{\prime}=h_{3}+h_{4} a^{2} .
\end{aligned}
$$

Next, this paper focuses on equation (21a). Let

$$
z(a) \stackrel{\text { def }}{=} h_{1} a+h_{2} a^{3}=0 .
$$

Two solution curves intersecting at $(0,0)$ can thus be acquired:

$$
\begin{aligned}
& a=0, \\
& a=\sqrt{-\frac{h_{1}}{h_{2}} .}
\end{aligned}
$$

The abovementioned equations provide dots and circles in polar coordinates, which correspond, respectively, to the equilibrium point and the limit cycle of the two-dimensional system of equations (21a) and (21b). Next, the stability is considered. Let

$$
z^{\prime}(a)=\frac{d z}{d a}=h_{1}+3 h_{2} a^{2} .
$$

For the trivial solution $a=0: z^{\prime}(0)=h_{1}$, when $h_{1}>0,(0$, $0)$ is unstable; when $h_{1}<0, a=0$ is asymptotically stable.

For the nontrivial solution $a=\sqrt{-h_{1} / h_{2}}: z^{\prime}\left(\sqrt{-h_{1} / h_{2}}\right)=$ $-2 h_{1}$, when $h_{1}>0$ the system has an asymptotically stable solution. However, when $h_{1}<0$, the system has an unstable solution. The former case corresponds to supercritical pitchfork bifurcation, and the latter subcritical pitchfork bifurcation.

Magnetorheological damping drops slowly with increasing temperature. When damping of the quasi-zerostiffness isolator is positive, the system has a trivial solution: $a=0$. Assuming that damping can be reduced to a negative value, a nontrivial solution appears for the system $a=\sqrt{-h_{1} / h_{2}}$ to form a limit cycle. In the nontrivial solution zone, on account of the constant expansion of the limit cycle, the vibration state that originally approached the stable focus tends to be divergent. Accordingly, the stability of the vibration system changes.

\section{Numerical Analysis}

The related parameters of the designed quasi-zero-stiffness vibration isolator are listed as follows: $m=75 \mathrm{~kg}, k=200,000 \mathrm{~N} /$ $\mathrm{m}, \delta=90,000 \mathrm{~N} / \mathrm{m}$, and $\xi=0.1 \mathrm{Ns} / \mathrm{m}$. During the vibration, the temperature of the magnetorheological damper rises, while damping decreases with time and finally stabilizes. The variation of damping can be expressed via the following function:

$$
c(\tau)=50-r \tau^{2}
$$

Here, $r$ is a parameter to describe the change of damping with temperature. Specifically, $c(\tau)>0$.

\subsection{Analysis of Vibration Characteristics before Any Change in} Damping. According to equation (16), the maximum excitation acceleration corresponding to no bifurcation under a stable state is $f_{\text {stable }}=2 w h_{5 \_ \text {stable }}=10.88 \mathrm{~m} / \mathrm{s}^{2}$. When the excitation acceleration exceeds this maximum value, the amplitude frequency curve appears.

As shown in Figure 2, when the excitation acceleration is below $f_{\text {stable }}$, each frequency in the frequency response curve corresponds to an amplitude, and the amplitude frequency curve, as the frequency sweeps downward, is identical with the curve as the frequency sweeps upward. When the excitation acceleration exceeds $f_{\text {stable, a jump occurs in the frequency }}$ response curve. As the frequency sweeps upward, the amplitude increases and jumps vertically to a corresponding low point upon arriving at the maximum and then drops gradually with increasing frequency. As the frequency sweeps downward, the amplitude increases gradually and jumps vertically to a corresponding high point when arriving at the inflection point and then decreases with decreasing frequency. In other words, a bifurcation-induced jump of the excitation frequency can be found in the frequency response curve. The amplitude frequency curves are different as the frequency sweeps downward or upward. As the excitation frequency increases, the vibration amplitudes in all frequency bands increase, and the unstable frequency band expands.

Figures 3 and 4 show the frequency response curves for $f=25 \mathrm{~m} / \mathrm{s}^{2}$. In Figure 3, $\delta=90,000 \mathrm{~N} / \mathrm{m}$, while in Figure 4 


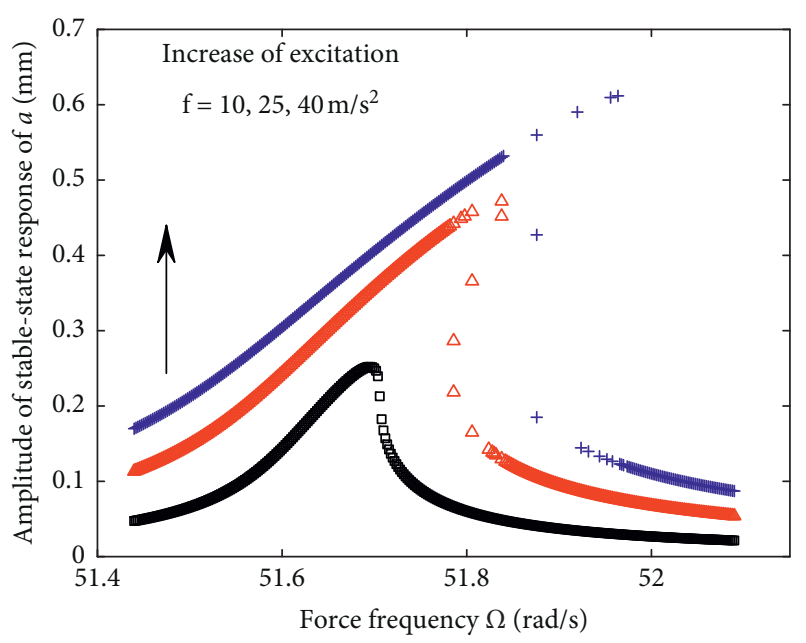

FIGURE 2: Effect of excitation acceleration on the stable vibration solution.

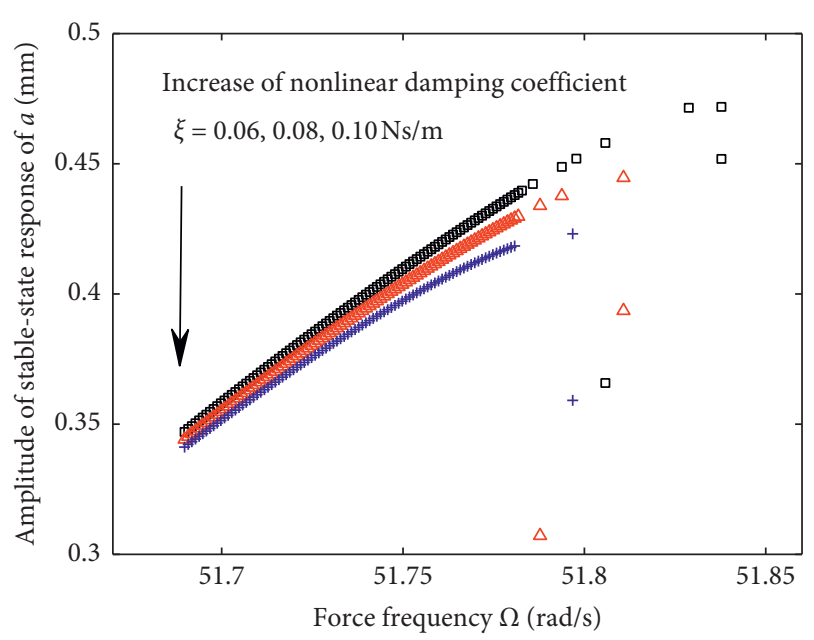

Figure 3: Effect of nonlinear damping on the stable vibration solution.

$\xi=0.1 \mathrm{Ns} / \mathrm{m}$. According to Figure 3, the unstable frequency band occupies a larger area at a smaller damping coefficient. Unlike Figure 2, as the damping coefficient drops, only the amplitude around the resonance frequency band increases, while the amplitudes of the other frequency bands remain almost unchanged. This behavior suggests that the change of the damping coefficient increases the amplitude around the resonant frequency point and expands the unstable frequency band and that it imposes slight effect on the vibration of the frequency band far away from the resonance point. The increase of nonlinear stiffness (Figure 4) does not lead to the vibration amplitude increase, but shifts the resonance point towards the right, namely, the unstable frequency band appears.

Figures 5 and 6 illustrates the results, assuming nonlinear stiffness $\delta_{s}=90,000 \mathrm{~N} / \mathrm{m}$ and nonlinear damping $\xi_{s}=0.1 \mathrm{Ns} / \mathrm{m}$, respectively. Based on Figure 3 analysis, the vibration amplitude decreases as the damping coefficient increases, accompanied by fewer solutions in the unstable

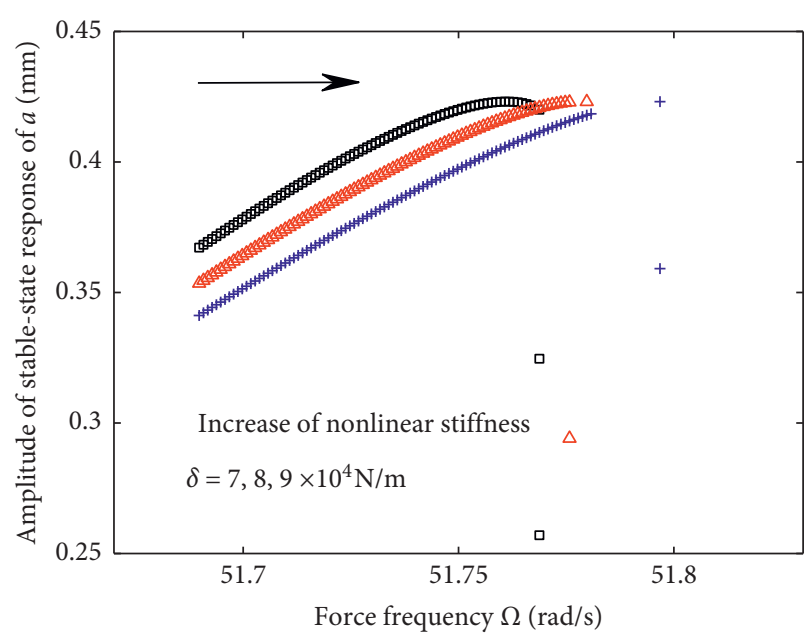

FIGURE 4: Effect of nonlinear stiffness on the stable vibration solution.

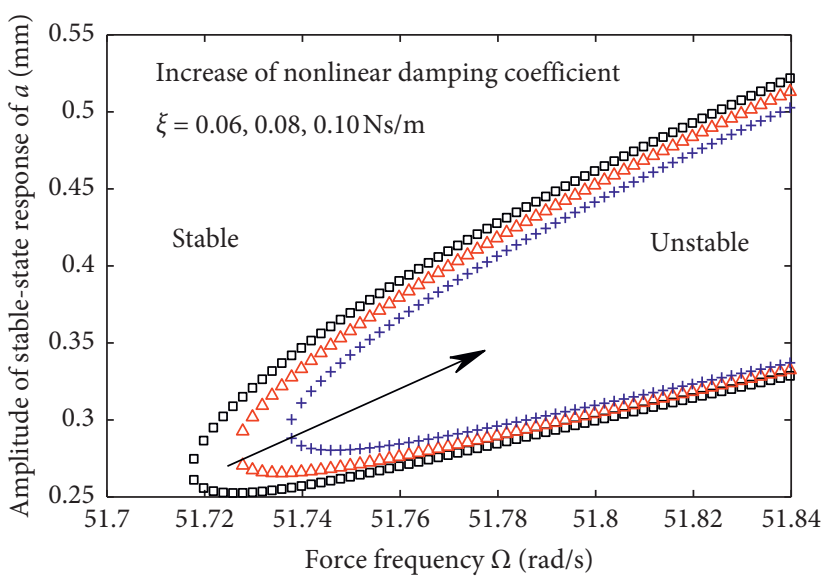

FIGURE 5: Effect of nonlinear damping on the solution's stability region.

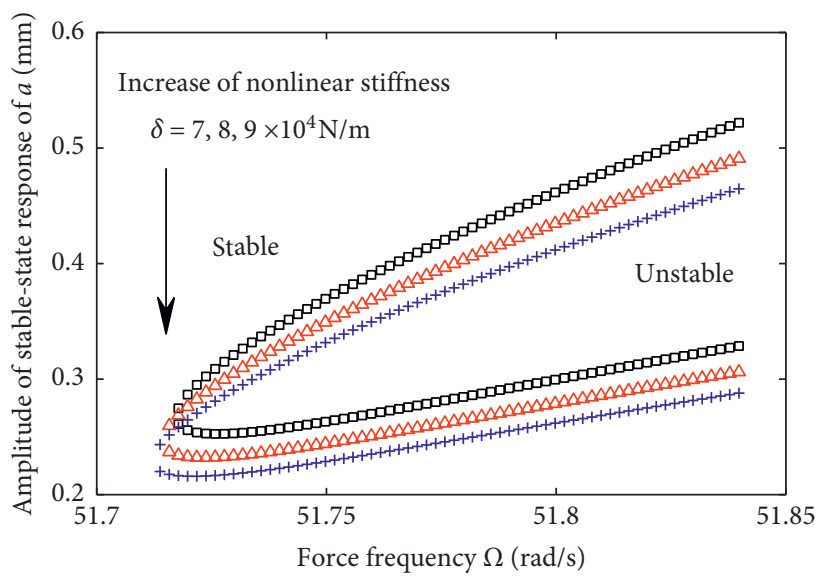

FIGURE 6: Effect of nonlinear stiffness on the solution's stability region.

region and enhanced system stability. As shown in Figure 5, when the nonlinear damping coefficient increases, the unstable region decreases along the direction of the saddle 


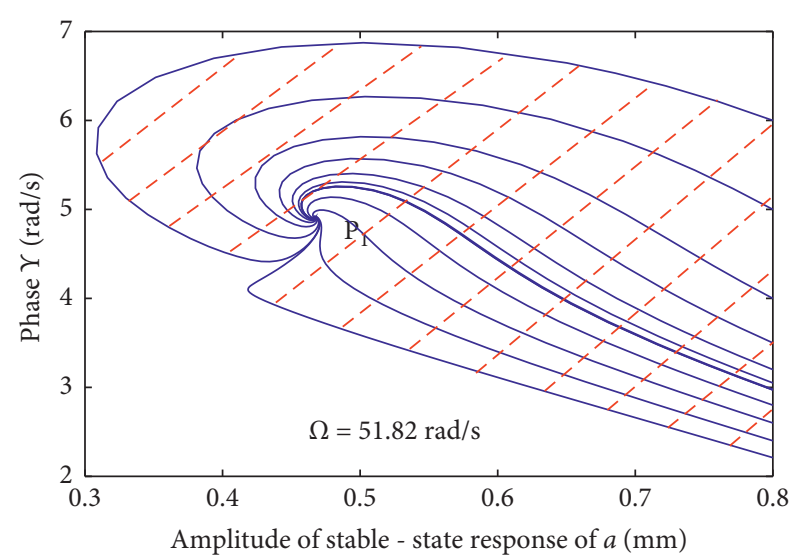

(a)

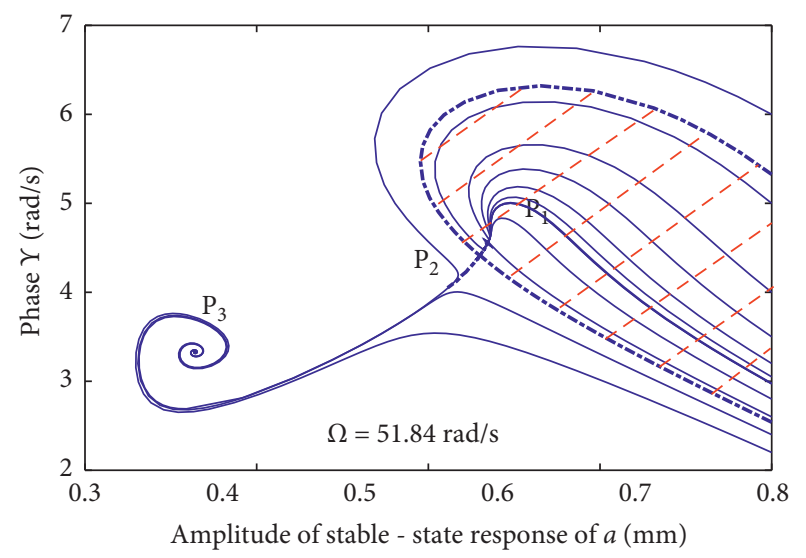

(c)

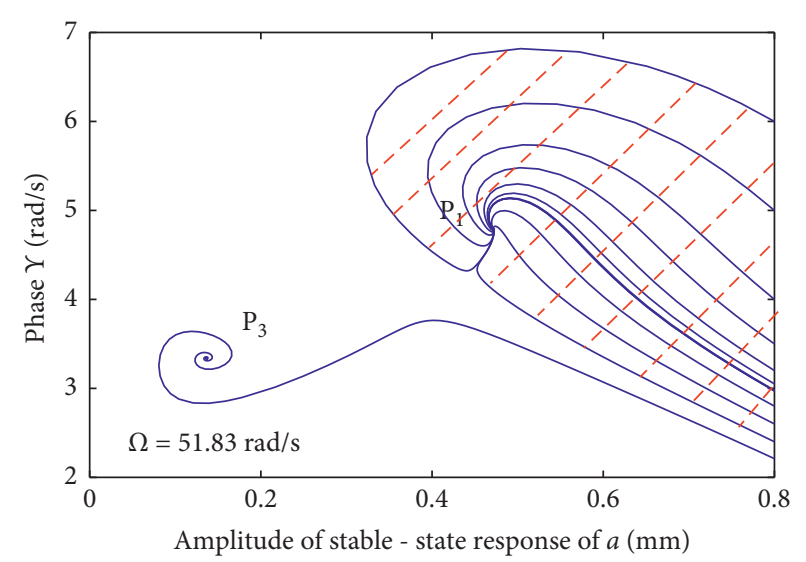

(b)

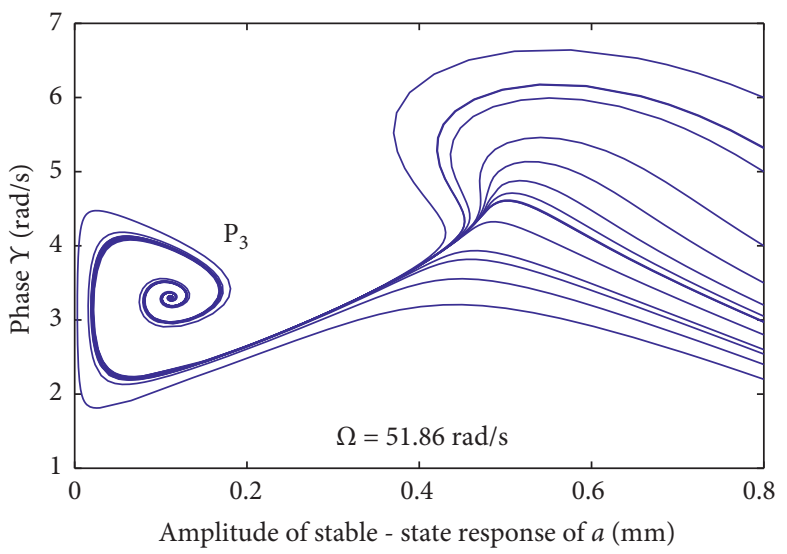

(d)

Figure 7: Variations of phase diagrams for different excitation frequencies: (a) $51.82 \mathrm{rad} / \mathrm{s}$, (b) $51.83 \mathrm{rad} / \mathrm{s}$, (c) $51.84 \mathrm{rad} / \mathrm{s}$, and (d) $51.86 \mathrm{rad} / \mathrm{s}$.

node. As described above, based on Figure 4, the peak vibration amplitude in the amplitude frequency curve moves rightward as the nonlinear stiffness increases. In addition, the number of solutions that fall in the unstable region increases, and the system stability weakens. As shown in Figure 6, with the increase of nonlinear stiffness, the unstable region as a whole moves downward and also expands gradually.

This study assumed that $w_{\text {down }}$ and $w_{\text {upper }}$ are the bifurcation critical upper-limit and lower-limit frequencies, respectively. As the excitation frequency $\Omega$ approaches $w_{\text {down }}$, the vibrations under all initial conditions are attracted to the stable focus $P_{1}$; once $\Omega>w_{\text {down }}$, another stable focus $P_{3}$ appears (see Figure 7(a) and Figure 7(b)). According to Figure $7(\mathrm{c})$, when $w_{\text {down }}<\Omega<w_{\text {upper }}$, stable focus $P_{1}$ and $P_{3}$ as well as the saddle-node $P_{2}$ can be seen. Only the initial value under certain conditions can form the trajectory that arrives at the saddle node $P_{2}$. The system stability, once slightly disturbed, will be attracted to $P_{1}$ or $P_{3}$. The dashed line in Figure $8(\mathrm{c})$ represents the boundary between two regions, the region outside the dashed line representing the states attracted to the stable focus $P_{3}$, while the other region representing the states attracted to the stable focus $P_{1}$. As the excitation frequency increases further and when $\Omega>w_{\text {upper }}$, the focus point $P_{1}$ disappears, and all states are attracted to the focus $P_{3}$, and the region, enclosed by a red dashed line, shrinks and disappears finally. All these phenomena indicate that upper and lower solutions of the amplitude frequency curves are asymptotically stable, while the intermediate solution is unstable. Since only asymptotic stability can be achieved in practical applications, jumping occurs. The abovementioned analysis results fit well with equation (24).

\subsection{Analysis of the Vibration Characteristics during Damping} Changing Process. During the vibration, rising temperature and decreasing damping characterize the magnetorheological damper.

According to equation (24), no limit cycle appears in the system vibration for the positive damping coefficient. Figure 9 shows the amplitude frequency response curves, which suggests that time-varying damping can induce a changing amplitude frequency response. Since the power spectrum density (PSD) of the vibration is proportional to the amplitude frequency response, it also explains that time-varying damping triggers the leakage of vibration power spectra, the decline of the corresponding linear spectral peak, the expansion of the frequency band, and less clear linear characteristics. The small box in Figure 9 highlights the leakage in detail. It is also shown in the figure that the vibration power 


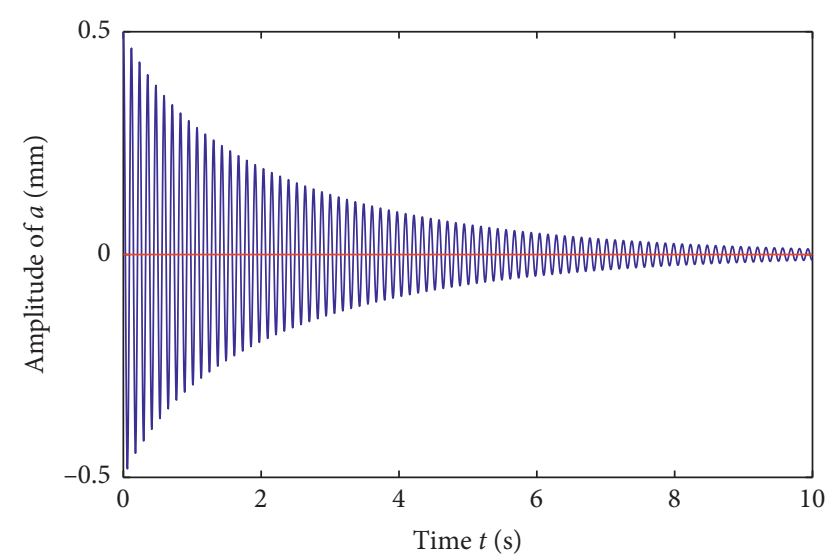

(a)

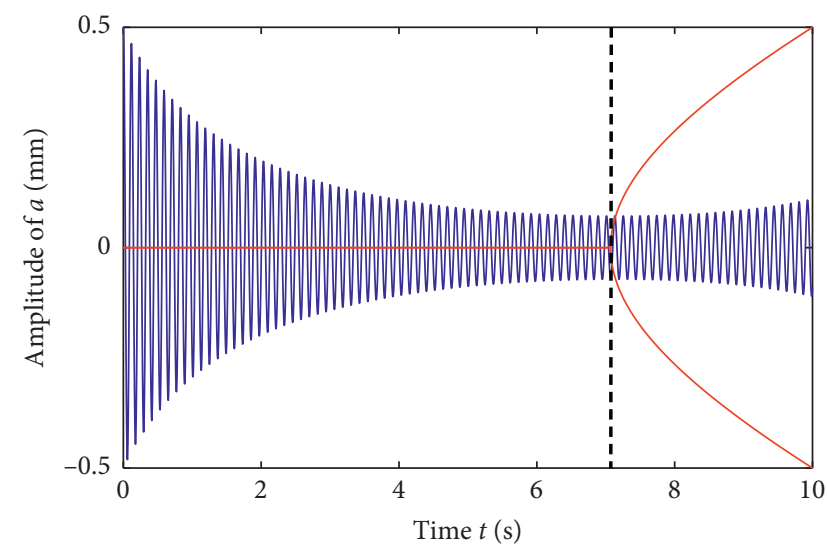

(c)

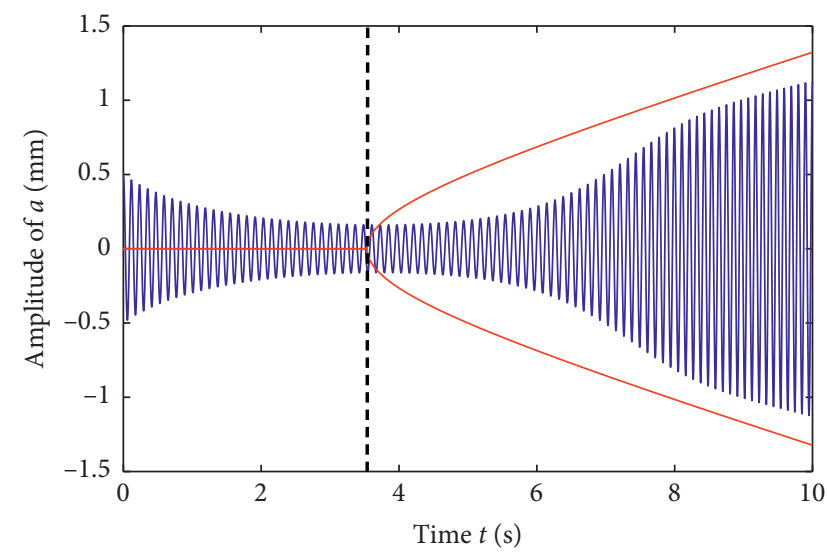

(e)

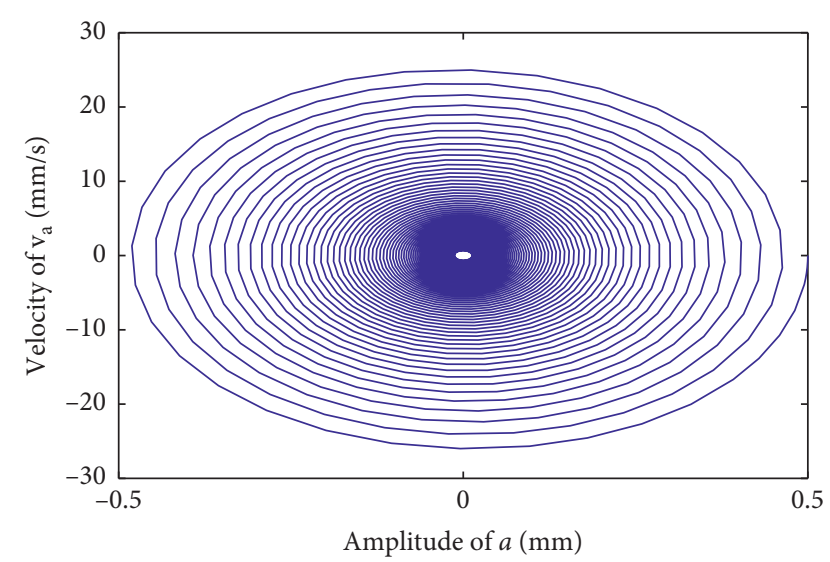

(b)

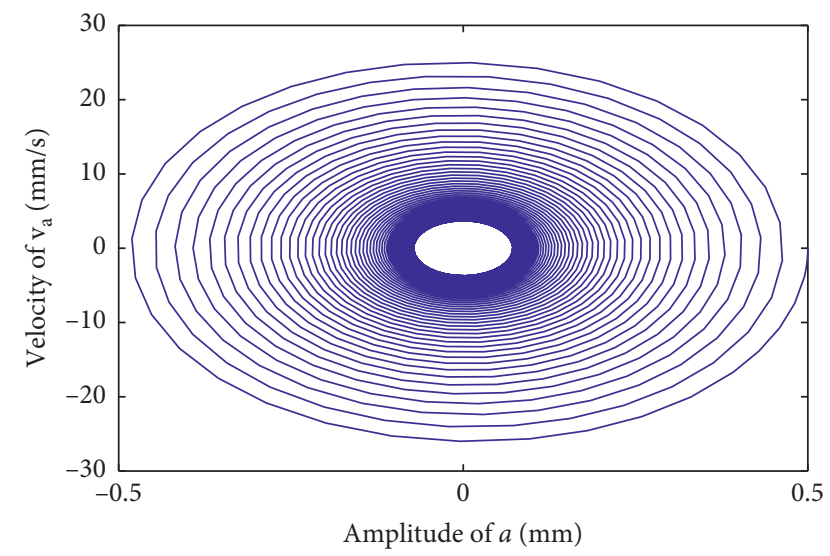

(d)

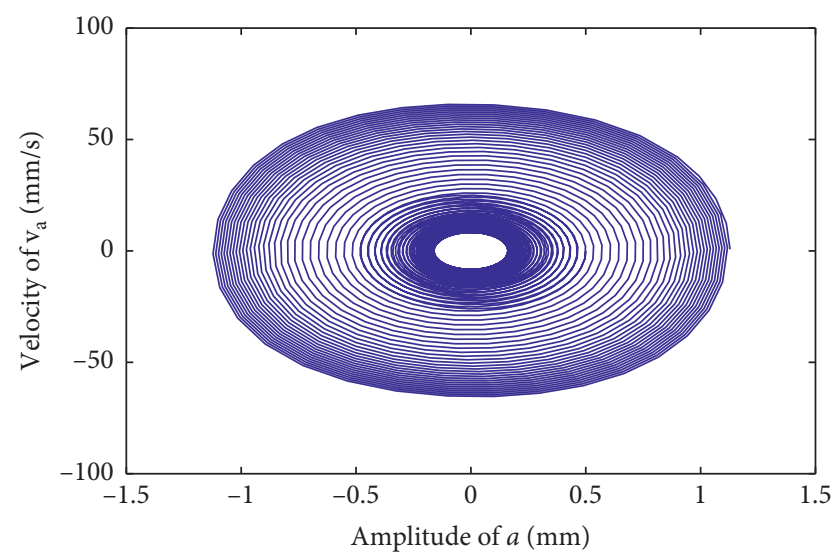

(f)

Figure 8: Time domain and phase diagrams: (a) $r=7$, (b) $r=7$, (c) $r=400$, (d) $r=400$, (e) $r=700$, and (f) $r=700$.

leakage becomes more obvious as the damping changes more rapidly and vice versa.

Assuming that the damping coefficient can be negative, the vibration time domain and phase diagrams at different changing rates are plotted (see Figure 8 ). When the damping changes slowly $(r=7)$ and still higher than 0 , the system vibration tends to be stable and no limit cycle can be observed, see Figures $8(\mathrm{a})$ and $8(\mathrm{~b})$. When damping changes substantially and becomes negative after certain time, a limit cycle appears in the system. As shown in Figures 8(c) and 8(e), damping on the left side of the dashed line is positive, while damping on the right side is negative. Limit cycles appear in Figures 8(d) and 8(f). Moreover, from Figure 8(c) and $8(e)$, we know the minimum amplitudes at different damping changing rates are different and the minimum amplitude is larger at a larger rate. After the damping becomes negative, the limit cycle forms and the vibration approaches the limit cycle. The gradual expansion of the limit cycle results in the divergence of vibration, but the vibration never exceeds the limit cycle. By comparing 


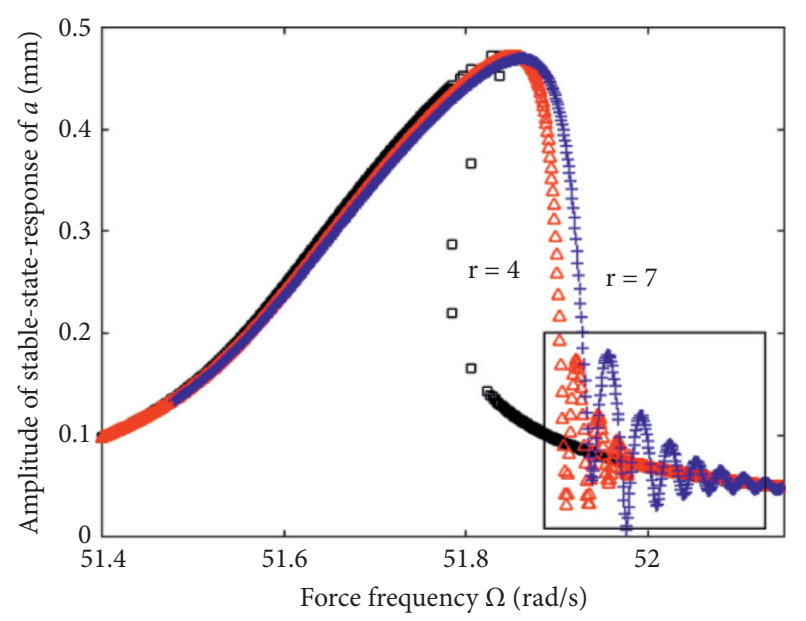

Figure 9: Amplitude frequency responses at different rates of damping change.

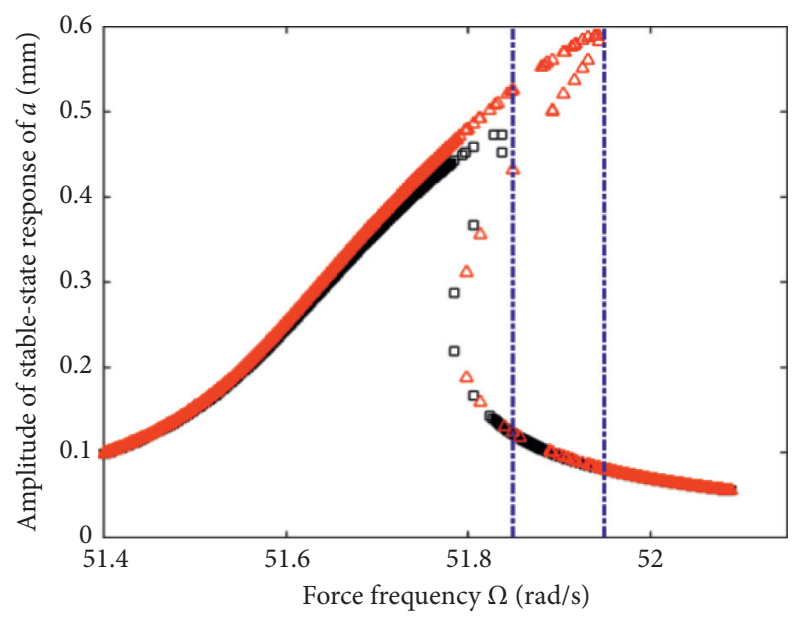

Figure 10: Amplitude frequency responses before and after damping changes.

Figures $8(\mathrm{a}), 8(\mathrm{c})$, and $8(\mathrm{e})$, due to different damping change rates with a same period of time, the initially identical vibration finally undergoes different changes: one tends to be stable and the other becomes divergent.

4.3. Analysis of the Vibration Characteristics after the Damping Change Stabilizes. During the vibration process, heat production and heat dissipation of the damper eventually reach a dynamic equilibrium. Damping stabilizes when temperature reaches a certain value. Although external bearing mass, excitation amplitude, and excitation frequency remain unchanged, damping changes. Therefore, the final stable state of some initial vibrations changes. As the damping changed from 50 to $20 \mathrm{Ns} / \mathrm{m}$, the vibration state in the intermediate frequency band, within the blue dashed lines in Figure 10, also varies.

Figure 11 shows the vibration states, before and after the damping changes, which is what is within the dashed lines in Figure 10. At the damping of $50 \mathrm{Ns} / \mathrm{m}$, the quasi-zerostiffness vibration isolator has only one solution and a stable

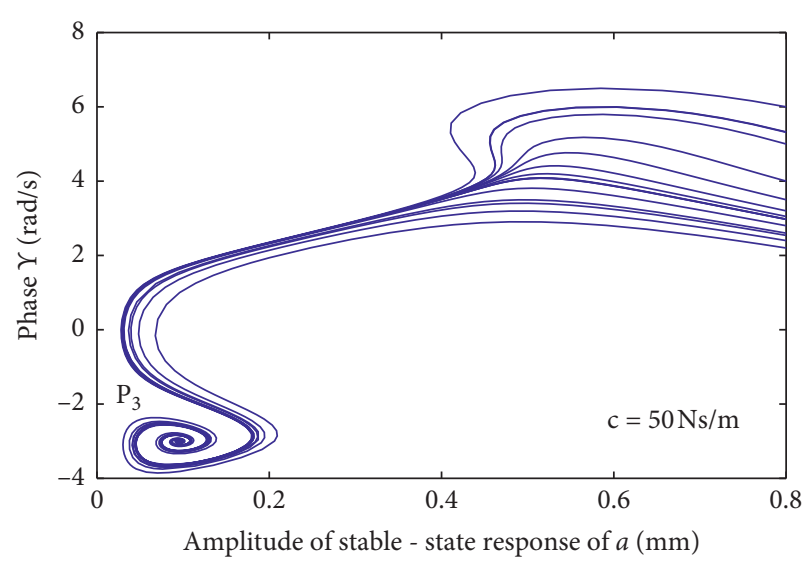

(a)

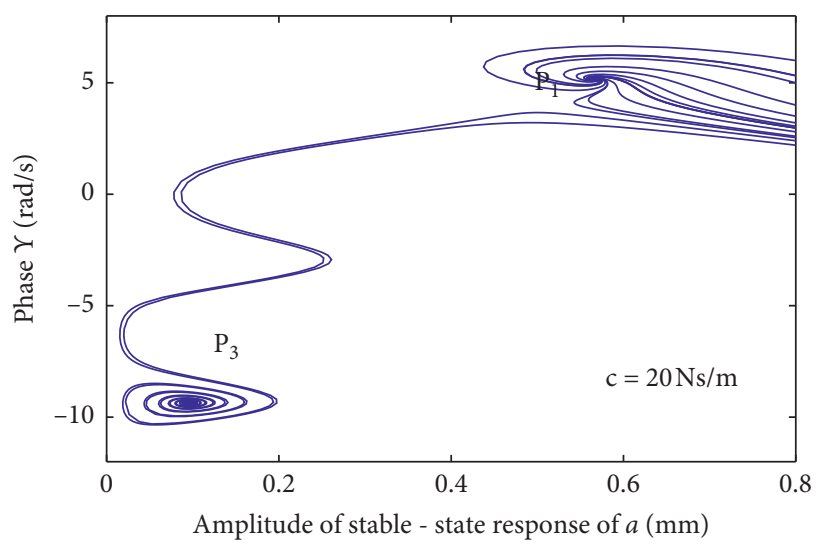

(b)

Figure 11: Stable focus before and after damping changes: (a) $c=50 \mathrm{Ns} / \mathrm{m}$ and (b) $c=20 \mathrm{Ns} / \mathrm{m}$.

vibration state. As the damping drops to $20 \mathrm{Ns} / \mathrm{m}$, the quasizero-stiffness vibration isolator has three solutions, and the solution in/on the intermediate branch is unstable, i.e., there are two possibilities for the vibration's stable state. This indicates that time-varying damping induces the change of the system's vibration state.

\section{Conclusions}

This study analyzed the effect of time-varying damping on the vibration characteristics of a quasi-zero-stiffness vibration isolator, by establishing a nonlinear vibration equation and solving this equation with a multiscale method. The following main conclusions can be drawn:

(a) In a system with quasi-zero-stiffness vibration isolators, the smaller nonlinear damping or the higher nonlinear stiffness or the higher excitation amplitude, the wider unstable frequency band and the larger unstable region. This results in weakened system vibration stability.

(b) Time-varying damping is equivalent to the stiffness term of the vibration system in terms of effect. Because of the stiffness change, the amplitude of the main resonance peak drops, accompanied by the 
leakage of energy and the less clear linear spectral characteristics. After the temperature stabilizes at a certain value, the vibration characteristics resume.

(c) Assuming that damping drops and turns negative, its change rate affects the final stable state. At a greater damping-change rate, the minimum vibration amplitude tends to be bigger. Furthermore, the final vibration state, which originally would approach stabilization, tends to be divergent.

(d) Time-varying damping reduces the vibration stability of the quasi-zero-stiffness vibration isolator. Under the condition of changing damping, the number of the final stable focus points, which correspond to the same initial vibration state, increases from 1 to 2 , and the system stability declines.

\section{Data Availability}

No data were used to support this study.

\section{Disclosure}

The authors would like to declare, on behalf of co-authors, that the work described was original research which has not been published previously and is not under consideration for publication elsewhere, in whole or in part.

\section{Conflicts of Interest}

The authors declare that they have no conflicts of interest.

\section{Authors' Contributions}

All listed authors have approved the manuscript.

\section{References}

[1] J. Zhou, X. Wang, D. Xu, and S. Bishop, "Nonlinear dynamic characteristics of a quasi-zero stiffness vibration isolator with cam-roller-spring mechanisms," Journal of Sound and Vibration, vol. 346, pp. 53-69, 2015.

[2] Y. Han, Q. Cao, and J. Ji, "Nonlinear dynamics of a smooth and discontinuous oscillator with multiple stability," International Journal of Bifurcation and Chaos, vol. 25, no. 13, Article ID 1530038, 2015.

[3] A. R. Valeev, "Vibration isolators for oil- and gas-transfer equipment with a low vibration frequency," Chemical and Petroleum Engineering, vol. 47, no. 5-6, pp. 374-377, 2011.

[4] C.-C. Lan, S.-A. Yang, and Y.-S. Wu, "Design and experiment of a compact quasi-zero-stiffness isolator capable of a wide range of loads," Journal of Sound and Vibration, vol. 333, no. 20, pp. 4843-4858, 2014.

[5] C. Cheng, S. Li, Y. Wang, and X. Jiang, "Resonance response of a quasi-zero stiffness vibration isolator considering a constant force," Journal of Vibration Engineering \& Technologies, vol. 6, no. 6, pp. 471-481, 2018.

[6] D. Xu, Q. Yu, J. Zhou, and S. R. Bishop, "Theoretical and experimental analyses of a nonlinear magnetic vibration isolator with quasi-zero-stiffness characteristic," Journal of Sound and Vibration, vol. 332, no. 14, pp. 3377-3389, 2013.
[7] D. Huang, W. Xu, W. Xie et al., "Dynamical properties of a forced vibration isolation system with real-power nonlinearities in restoring and damping forces," Nonlinear Dynamics, vol. 81, no. 1-2, pp. 641-658, 2015.

[8] H. Li and J. Zhang, "Design and analysis of a magnetic QZS vibration isolator," Applied Mechanics and Materials, vol. 470, pp. 484-488, 2014.

[9] I. Kovacic, M. J. Brennan, and T. P. Waters, "A study of a nonlinear vibration isolator with a quasi-zero stiffness characteristic," Journal of Sound and Vibration, vol. 315, no. 3, pp. 700-711, 2008.

[10] L. Meng, J. Sun, and W. Wu, "Theoretical design and characteristics analysis of a quasi-zero stiffness isolator using a disk spring as negative stiffness element," Shock and Vibration, vol. 2015, Article ID 813763, , 2015.

[11] T. Yang and Q. Cao, "Nonlinear transition dynamics in a time-delayed vibration isolator under combined harmonic and stochastic excitations," Journal of Statistical Mechanics: Theory and Experiment, vol. 2017, Article ID 043202, 2017.

[12] Y. Araki, K. Kimura, T. Asai, T. Masui, T. Omori, and R. Kainuma, "Integrated mechanical and material design of quasi-zero-stiffness vibration isolator with superelastic $\mathrm{Cu}$ Al-Mn shape memory alloy bars," Journal of Sound and Vibration, vol. 358, pp. 74-83, 2015.

[13] Y. Liu, L. Xu, C. Song, H. Gu, and W. Ji, "Dynamic characteristics of a quasi-zero stiffness vibration isolator with nonlinear stiffness and damping," Archive of Applied Mechanics, vol. 89, no. 9, pp. 1743-1759, 2019.

[14] C. Cheng, S. Li, Y. Wang, and X. Jiang, "Force and displacement transmissibility of a quasi-zero stiffness vibration isolator with geometric nonlinear damping," Nonlinear Dynamics, vol. 87, no. 4, pp. 2267-2279, 2016.

[15] M. Amabili, "Nonlinear damping in large-amplitude vibrations: modelling and experiments," Nonlinear Dynamics, vol. 93, no. 1, pp. 5-18, 2017.

[16] S. M. M. Mofidian and H. Bardaweel, "Displacement transmissibility evaluation of vibration isolation system employing nonlinear-damping and nonlinear-stiffness elements," Journal of Vibration and Control, vol. 24, no. 18, pp. 4247-4259, 2017.

[17] S. Sun, X. Tang, J. Yang et al., "A new generation of magnetorheological vehicle suspension system with tunable stiffness and damping characteristics," IEEE Transactions on Industrial Informatics, vol. 15, no. 8, pp. 4696-4708, 2019.

[18] Y. H. Guo, E. W. Chen, Q. Wu, Y. M. Lu, and Z. Q. Xia, "The calculation of the equivalent linear damping coefficient of the magnetorheological damper," Applied Mechanics and Materials, vol. 336-338, pp. 475-479, 2013.

[19] M. McKee, X. Wang, and F. Gordaninejad, "Effects of temperature on performance of a compressible magnetorheological fluid damper-liquid spring suspension system," Active and Passive Smart Structures and Integrated Systems, vol. 2011, no. 7977, Article ID 797712, 2011.

[20] P. Mitrouchev, A. Klevinskis, V. Bucinskas et al., "Analytical research of damping efficiency and heat generation of magnetorheological damper," Smart Materials and Structures, vol. 26, Article ID 065026, 2017.

[21] J. Maroofi and S. H. Hashemabadi, "Experimental and numerical investigation of parameters influencing anisotropic thermal conductivity of magnetorheological fluids," Heat and Mass Transfer, vol. 55, no. 10, pp. 2751-2767, 2019.

[22] S. Chen, J. Huang, K. Jian et al., "Analysis of influence of temperature on magnetorheological fluid and transmission 
performance," Advances in Materials Science and Engineering, vol. 2015, Article ID 583076, 2015.

[23] D. M. Wang, Y. F. Hou, and Z. Z. Tian, "A novel high-torque magnetorheological brake with a water cooling method for heat dissipation," Smart Materials and Structures, vol. 22, Article ID 025019, 2013.

[24] S. R. Patil, K. P. Powar, and S. M. Sawant, "Thermal analysis of magnetorheological brake for automotive application," Applied Thermal Engineering, vol. 98, pp. 238-245, 2016.

[25] R. Russo and M. Terzo, "Design of an adaptive control for a magnetorheological fluid brake with model parameters depending on temperature and speed," Smart Materials and Structures, vol. 20, Article ID 115003, 2011.

[26] J. Cazenove, D. A. Rade, A. M. G. Lima et al., "A numerical and experimental investigation on self-heating effects in viscoelastic dampers," Mechanical Systems and Signal Processing, vol. 27, pp. 433-445, 2012.

[27] G. T. Ngatu, C. S. Kothera, W. Hu et al., "Effects of temperature on magnetorheological fluid elastomeric lag dampers for helicopter rotors," 50th AIAA/ASME/ASCE/AHS/ASC structures," Structural Dynamics, and Materials Conference, vol. 17, pp. 1-16, 2009.

[28] V. P. Mikhailov, A. M. Bazinenkov, P. A. Dolinin, and G. V. Stepanov, "Research on the dynamic characteristics of a controlled magnetorheological elastometer damper," Instruments and Experimental Techniques, vol. 61, no. 3, pp. 427432, 2018.

[29] Y. Zhong, J. Tu, Y. Yu, J. Xu, and D. Tan, “Temperature compensation in viscoelastic damper using magnetorheological effect," Journal of Sound and Vibration, vol. 398, pp. 39-51, 2017. 